\title{
Various approaches of molecular process for diagnosis of viral disease
}

\begin{abstract}
The emergence and re-emergence of various viral diseases indicate the need for the development of effective and authentic new diagnostic methods. The diagnostic application of gel-based PCR, general PCR systems, phylogeny, molecular epidemiology, real-time PCR, amplification without thermo-cycling, multiplex PCR, nucleic acid extraction and pipetting robotics, automation and quality control, including internal controls plays significant role for laboratory diagnosis of viral disease. The development of various probes and microarrays, as well as ultra rapid PCR and sequencing methods is further improving the arsenal of nucleic acid based molecular diagnosis for viruses. Further trends of diagnostic development are also mentioned, in order to combat viral infections more effectively in the future.
\end{abstract}

Volume 4 Issue 5 - 2017

\author{
Ravikant, Satish Gupte, Maninder kaur, \\ Mandeep kaur \\ Department of Microbiology, Gian Sagar Medical College \& \\ Hospital, India
}

Correspondence: Satish Gupte, Department of Microbiology, Gian Sagar Medical College \& Hospital, India,

Email drsatishgupte@hotmail.com

Received: March 18, 2017 | Published: May 01, 2017

Keywords: amplification techniques, automation, viral diseases, molecular methods

\section{Introduction}

Isolation and identification of causative agent always help the physician in control and prevention of infectious disease. Molecular diagnosis as a more sensitive and specific method, has become the best option to investigate various microbes, including viruses. Advances in scientific and technological approaches resulted in reforming diagnostic microbiology. Many new users' friendly commercial assays have been developed for this purpose. ${ }^{1}$ In the current scenario, remarkable efforts have been made for genomes sequencing of all medically important microbes. Investigation of nucleic acids in the detection of variety of organisms related to infectious diseases such as nosocomial infections, sexually transmitted diseases, and immune-compromised host infections. ${ }^{2,3}$ Nucleic acid detection - Detection of virus encoded DNA and RNA is done with polymerase chain reaction. Nucleic acid hybridization with virus-specific probes detects specific viruses. Molecular techniques are usually used to confirm positive serological results due to their higher sensitivity and specificity. Variations of PCR such as nested reverse transcriptase PCR and real time PCR can also be used to determine viral loads in patient serum. This is often used to monitor treatment success in HIV cases. Gene secquencing Some viruses require specialized gene sequencing techniques to be carried out in order to help predict the type of infection that will display in the patient. Other application of molecular techniques is in epidemiological and clinical investigations for viral infections such as HPV (Human Papilloma Virus) in cervical and genotyping for HCV (Hepatitis $\mathrm{C}$ virus). ${ }^{4,5}$

Some of advances that include the use of real-time measurements, isothermal amplification, and the inclusion of internal quality assurance protocols, device miniaturization and the automation of specimen processing in totality with molecular methods always give an edge over conventional methods of viral detection. And molecular assays are currently used for diagnostics, antiretroviral monitoring and drug resistance characterization in developed countries. Recent approaches such as microarrays and next- generation sequencing are expected to have a huge impact in the next revolution of pathogen identification. In the context there is no need for a molecular diagnostic method to support the replication of an organism. The most necessity to identify a specific molecule in a reaction is a proper molecular probe. Nucleic acid amplification technology has reduced the time of viral detection and characterization. ${ }^{6}$ Here, we focused on the most recently molecular approaches in diagnosis of viral infection and their characteristics.

\section{Non amplified nucleic acid probes}

Nucleic acid probes are small fragments of Deoxyribonucleic acid or ribonucleic acid that have being labeled with enzymes, chemiluminescent molecules or radioisotopes. Complementary sequences are targets for these in samples may be carried out on liquid- phase, solid-phase and in situ hybridization. Due to poor analytical sensitivity, these techniques might be used only to detection a large scale microorganisms. ${ }^{5,6}$ Regardless of advances in this technology, there are number of challenges in application of viral testing related to precision, accuracy and standardization. HPV and CMV (Cytomegalovirus) can be detected by using these probes.

\section{Amplified nucleic acid techniques}

Polymerase chain reaction is the most commonly applied qualitative diagnostic method. Nucleic acid testing for infectious diseases is almost completely performed in centralized laboratories using highend instrumentation and expert personnel. Other methodologies which are not PCR based techniques are based on amplification of signal, target or probes. One of these methods is NASBA (nucleic acid sequence-based amplification. ${ }^{7}$ This is a strong amplification method which has been used to identify a number of pathogens, including RNA viruses such as HIV and HCV. Other viruses that can be detected are $\mathrm{HCV}, \mathrm{HBV}, \mathrm{HPV}, \mathrm{CMV}, \mathrm{VZV}$, BK virus, HIV-1, Influenza A and $\mathrm{B}$, and $\mathrm{HSV}$.

\section{Microarrays}

ADNA microarray (also commonly known as DNA chip or biochip) is a collection of microscopic DNA spots attached to a solid surface. Scientists use DNA microarrays to measure the expression levels of large numbers of genes simultaneously or to genotype multiple regions of a genome. Each DNA spot contains picomoles (10-12 moles) of a specific DNA sequence, known as probes (or reporters or oligos). These can be a short section of a gene or other DNA element 
that are used to hybridize a cDNA or cRNA (also called anti-sense RNA) sample (called target) under high-stringency conditions. Probetarget hybridization is usually detected and quantified by detection of fluorophore, silver, or chemiluminescence-labeled targets to determine relative abundance of nucleic acid sequences in the target.

DNA microarrays can be used to detect DNA (as in comparative genomic hybridization), or detect RNA (most commonly as cDNA after reverse transcription) that may or may not be translated into proteins. Used for identification of Respiratory viruses, HCV, HPV, HIV, HSV and SARS. ${ }^{8}$ Since many DNA sequences can be present on a slide, concurrent testing for several viruses is possible for microarray analysis for instance, to study polymorphisms of the HIV protease gene for detection of drug resistance

\section{Multiplex methods: nucleic acid-based}

Nucleic acid-based - Multiplexing technologies enable the simultaneous identification of multiple viruses in a single assay which can significantly decrease the time, cost and labor-input related to conventional single reaction detection methods.

Multiplexed Microsphere - Based Array Microsphere-based suspension array technologies present a stage for nucleic acid identification which have some advantages as well as rapid data achievement, high specificity and sensitivity and multiplexed analysis potential. ${ }^{5,6}$ Compared to planar microarrays, advantages of these techniques are including being user friendly, higher statistical power, low cost, faster hybridization kinetics and more flexibility in preparation. A multiplexed assay for diagnosis of viral infection using this system has been developed for HCV, HSV, HIV and HPV typing with high specific outcome.

\section{Whole-genome sequencing}

Subsequent to electron microscopy, cell culture and Polymerase chain reaction, whole genome sequencing (WGS) is one of these techniques which are now changing the way of understanding viruses, mainly in the fields of genome sequencing, development, ecology, detection and transcriptomics. Whole genome sequencing is the basis for the inclusive understanding of an organism's function. Recent studies showed that characterization of complex mixture of microflora in environmental context can be done using high-throughput sequencing. Next- generation sequencing (NGS) techniques present an unique "step-change" raise in the quantity of sequence data which can be generated from a sample. ${ }^{8,9}$ Although this method used for de novo sequencing of large genomes, to achieve ultra deep coverage, NGS can be applied for resequencing of small viral genome. ${ }^{9}$ Thus, NGS potential gives information further than the consensus for a viral sample through revealing present nucleotide substitutions in just a small portion of the population such as sequencing of new influenza viruses, detection of viral genome inconsistency and evolution within the host, such as assessment of human immunodeficiency virus and human hepatitis $\mathrm{C}$ virus species, and monitoring of low- abundance antiviral drug-resistance mutations.

\section{Conclusion}

This period of viral investigation has begun which causes new challenges to realize the role of recently revealed viral diversity in both disease and health. A variety of molecular methods based on nucleic acid including amplified and non-amplified probes, microarrays, and target amplification are at the present applied for detection of organisms, their virulence factors and drug resistance determinants. The prospect of the molecular diagnostics of viral infections will indisputably be focused on a significant enhance in the number of information detected with significant basic, rapid platforms that will need complex software analysis to resolve the data for apply in clinical strategies. There is a need for special circumstances are such as modern equipments and trained personnel to enhance the efficacy of these new technologies in diagnostics.

\section{Acknowledgements}

None.

\section{Conflict of interest}

The author declares no conflict of interest.

\section{References}

1. Erwin S, Fred S. Advances in molecular diagnosis of HBV infection and drug resistance. Int J Med Sci. 2005;2(1):8-16.

2. Fernando C. Application of molecular diagnostic techniques for viral testing. Open Virol J. 2012;6:104-114.

3. Massimo C, Patrizia B. Are three generations of quantitative molecular methods sufficient in medical virology. New Microbiol. 2015;38(4):437441.

4. Debiasi RL, Tyler KL. Molecular methods for diagnosis of viral encephalitis. Clin Microbiol Rev. 2004;17(4):903-925.

5. Read SJ, Kurtz JB. Laboratory diagnosis of common viral infections of the central nervous system by using a single multiplex pcr screening assay. J Clin Microbiol. 1999;37(5):1352-1355.

6. Gulley ML. Molecular diagnosis of Epstein-Barr virus-related diseases. J Mol Diagn. 2001;3(1):1-10.

7. Gutiérrez-Xicoténcatl L, Plett-Torres T, Madrid-González CL, et al. Molecular diagnosis of human papillomavirus in the development of cervical cancer. Salud Publica Mex. 2009;51(Suppl 3):S479-S488.

8. Terezinha Maria de Paiva. Use of molecular test for adenovirus detection between different pediatric patients. Rev Paul Pediatr. 2015;33(2):134 135.

9. Beck ET, Henrickson KJ. Molecular diagnosis of respiratory viruses. Future Microbiol. 2010;5(6):901-916. 